\title{
Avaliação do uso de soros antivenenos na emergência de um hospital público regional de Vitória da Conquista (BA), Brasil
}

\author{
Evaluation of the use of antivenom sera in the emergency service of \\ a regional public hospital in Vitória da Conquista (BA), Brazil
}

Caroline Rocha Santana (https://orcid.org/0000-0002-4460-2412) ${ }^{1}$

Márcio Galvão Oliveira (https://orcid.org/0000-0001-5281-7889) ${ }^{1}$

${ }^{1}$ Instituto Multidisciplinar em Saúde, Universidade Federal da Bahia. R. Hormindo Barros 58, Candeias. 45029-094 Vitória da Conquista BA Brasil.carolinerochaufba@ gmail.com

\begin{abstract}
This study aims to characterize the epidemiological profile of victims of scorpion and snakebite envenomations and to evaluate the adequacy of antivenom sera prescriptions. This is a cross-sectional study whose data sources were the envenomation notification information sheets of the Notifiable Diseases Information System in the city of Vitória da Conquista (BA), Brazil. We included information on scorpion or snakebite envenomations attended in the municipality in the period between July 2016 and June 2017. The data obtained and the variables of interest were analyzed according to the questions of this study. In the observed period, 293 victims of envenomations were treated. Of these, 149 (50.9\%) were men, and $114(38.9 \%)$ were $20-59$ years old. In total, $235(80.9 \%)$ cases of scorpionism and $58(19.1 \%)$ of ophidism were reported. Of these, 203 (69.3\%) were classified as mild, and in 200 (68.5\%) cases, serum therapy was prescribed for the patients. Regarding the adequacy of the prescriptions, 172 (59.7\%) were considered inadequate, and of these, the use of some vials above than indicated was the most frequent. The inappropriate prescription of antivenom sera occurred in approximately $60 \%$ of the evaluated cases. Despite this, most accidents were classified as mild - in young men.

Key words Ophidic envenomation, Scorpion bites, Inappropriate use
\end{abstract}

Resumo O presente estudo tem como objetivo caracterizar o perfil epidemiológico de vítimas de acidentes escorpiônicos e ofídicos e avaliar a adequação das prescrições de soros antivenenos. Estudo transversal cujas fontes de dados foram as fichas de notificação de acidentes por animais peçonhentos do Sistema de Informação de Agravos de Notificação no município de Vitória da Conquista (BA), Brasil. Foram incluidas as informações de acidentes escorpiônicos ou ofídicos no período entre julho de 2016 e junho de 2017 atendidos no município. Os dados obtidos e as variáveis de interesse foram analisadas de acordo com as perguntas deste estudo. No periodo observado foram atendidas 293 vitimas de acidentes por animais peçonhentos. Destas, 149 (50,9\%) foram homens e 114 (38,9\%) possuíam entre 20 a 59 anos. Foram 235 (80,9\%) casos de escorpionismo e 58 (19,1\%) de ofidismo. Destes, 203 (69,3\%) foram classificados como leves e em 200 (68,5\%) casos foi prescrita soroterapia para estes pacientes. Quanto à adequação das prescrições, 172 (59,7\%) foram julgadas inadequadas e destas, o uso de número de ampolas acima do indicado foi a mais frequente. A prescrição inapropriada de soros antivenenos ocorreu em aproximadamente em $60 \%$ dos casos avaliados. Apesar disso, a maioria dos acidentes foi classificada como leve, em homens jovens.

Palavras-chave Acidente Ofídico, Picadas de escorpião, Uso inapropriado 


\section{Introdução}

O escorpionismo e o ofidismo são os principais acidentes por animais peçonhentos e estes eventos são considerados doenças negligenciadas pela Organização Mundial de Saúde ${ }^{1}$, especialmente em países tropicais e subtropicais e/ou em processo de desenvolvimento econômico ${ }^{2-6}$.

O escorpionismo destaca-se com mais de 1 milhão de casos reportados por ano. Oriente Médio, América do Sul, Índia, África e México figuram como áreas endêmicas de escorpiões perigosos à saúde humana ${ }^{6,7}$. No Brasil (com 90.922 casos notificados em 2016) ${ }^{8}$ as espécies Tityus stigmurus, Tityus serrulatus, Tityus bahiensis e Tityus cambridgei são as principais causadoras de acidentes por escorpiões ${ }^{7}$. O ofidismo, por sua vez, tem alta prevalência nas Américas Central e do Sul, e no Brasil, no ano de 2016, foram notificados mais de 26.000 casos $^{2,4,8,9}$. A maioria dos acidentes ofídicos brasileiros são por picadas de serpentes do gênero Bothrops (aproximadamente $90 \%$ dos casos notificados) e Crotalus $^{10}$.

Ofidismo e escorpionismo são condições que podem levar a complicações clínicas importantes, inclusive à morte ${ }^{2,11-16}$. As manifestações locais são as mais comuns, destacando-se dor, eritema e edema no local da picada. Mas as altas quantidades de venenos destes animais podem levar a manifestações sistêmicas graves como disfunção respiratória, renal, vascular, cardíaca e/ou neurológica ${ }^{4,7,11,17-20}$, sendo as crianças a população mais susceptível às complicações pós-envenenamento ${ }^{21-23}$.

O manejo de pacientes vítimas de escorpionismo ou de ofidismo é feito com suporte básico das condições vitais, associado ao tratamento sintomático e à soroterapia específica, quando necessária. A soroterapia antiescorpiônica só é indicada em casos de classificação "moderada", em crianças com idade menor que 10 anos e em todos os casos classificados como graves ${ }^{24}$. Na falta do soro antiescorpiônico pode ser utilizado o soro antiaracnídeo (que inclui anticorpos contra o veneno do Tityus $)^{25}$. No ofidismo o tratamento varia de acordo com o gênero da serpente, estando disponíveis no Brasil os soros: botrópico (para acidentes com serpentes do gênero Bothrops, como jararaca), crotálico (para picadas de Crotalus) e elapídico (para picadas pelas serpentes do gênero Micrurus, popularmente conhecidas como cobras corais). Existem ainda soros associados (botrópico-laquético e botrópico-crotálico) usados para situações em que o tipo de serpente é desconhecido, sendo o soro botrópi- co-laquético utilizado especialmente em acidentes que se suspeita que a serpente envolvida seja do gênero Lachesis (conhecida mais comumente como surucucu $)^{25-27}$.

Em 2016, o Ministério da Saúde brasileiro divulgou nota com orientações sobre a necessidade de adequações nas doses de antivenenos indicadas para tratamento por jararacas e escorpiões na tentativa de reduzir o desperdício, sem prejuízo para as vítimas de acidentes por animais peçonhentos $^{28}$. No entanto, diversos estudos têm demonstrado baixa adesão de profissionais de saúde às recomendações de diretrizes clínicas em diversas áreas ${ }^{29,30}$. O presente estudo tem como objetivo caracterizar o perfil epidemiológico de usuários vítimas de acidentes escorpiônicos e ofídicos e avaliar a adequação das prescrições de soros antivenenos no serviço de emergência de um hospital regional.

\section{Método}

Estudo transversal onde foram analisados dados secundários de acidentes por animais peçonhentos através do Sistema de Informação de Agravos de Notificação (SINAN) no município de Vitória da Conquista, Bahia.

O Hospital Geral de Vitória da Conquista (HGVC) é serviço de referência em acidentes com animais peçonhentos para a cidade de Vitória da Conquista e região circunvizinha (regiões de escorpiões do gênero Tityus e de serpentes do gênero Bothrops e Crotalus), atendendo diariamente vítimas de escorpionismo e ofidismo. Apenas o HGVC recebe os soros em Vitória da Conquista e quando os pacientes recorrem a outros serviços da cidade estes são transferidos para o HGVC ou os soros são dispensados e transportados para estes serviços mediante notificação de agravo.

Os dados foram coletados das fichas de notificações de acidentes por animais peçonhentos lançadas pelo setor de Vigilância Epidemiológica do HGVC no SINAN. Foram incluídas todas as notificações de acidentes por escorpião ou por serpente no período compreendido entre julho de 2016 e junho de 2017. Para a análise de adequação das prescrições dos soros antivenenos foram excluídas as notificações em que não havia sido descrito o tipo de acidente (ausência da identificação do animal peçonhento causador do acidente na ficha de notificação), classificação de gravidade, presença ou ausência de soroterapia.

Os critérios de adequação de uso dos soros foram construídos de acordo com a classificação de 
gravidade destes acidentes segundo informações dos protocolos de soroterapia brasileiros contidos no Manual de Diagnóstico e Tratamento de Acidentes por Animais Peçonhentos (2001) ${ }^{26}$ e no Guia de Vigilância em Saúde $(2014)^{25}$, atualizados pela Nota Informativa da Coordenação-Geral de Doenças Transmissíveis (2016) ${ }^{28}$. Os dados sobre tipo de acidente, classificação de gravidade, presença ou ausência de soroterapia, número de ampolas prescritas e tipo(s) de soro(s) utilizados foram confrontados com os critérios de adequação. Dessa forma, as soroterapias descritas foram classificadas como adequadas ou inadequadas. As inadequações observadas foram classificadas em: "uso de ampolas superior ao recomendado", "uso de ampolas inferior ao recomendado" e "uso de tipo de soro inadequado".

Os dados obtidos foram tabulados em banco de dados no Microsoft ${ }^{\circledR}$ Office Excel 2010 e analisados através do software IBM SPSS Statistics 20.0. Foram calculadas as medidas de tendência central, dispersão e frequências associadas às variáveis pertinentes, como dados individuais (sexo, idade, município de residência, raça/cor, escolaridade), dados epidemiológicos (zona de ocorrência, localidade de ocorrência do acidente, tempo decorrido entre a picada e o atendimento), dados clínicos (verificação da presença ou não de manifestações locais e sistêmicas, bem como quais apresentadas em caso de presença e classificação da gravidade do acidente) e dados sobre a terapêutica utilizada (presença ou ausência de soroterapia, número de ampolas prescritas e tipo(s) de soro(s) utilizados). As associações entre variáveis categóricas foram verificadas através do Teste de Qui-quadrado e considerou-se como diferença estatisticamente significativa quando a probabilidade foi menor do que $0,05(\mathrm{p}<0,05)$.

Este projeto de pesquisa foi aprovado pela Comitê de Ética em Pesquisa com Seres Humanos do Instituto Multidisciplinar em Saúde da Universidade Federal da Bahia.

\section{Resultados}

No período do estudo foram atendidas 293 vítimas de acidentes por animais peçonhentos. Destas, $149(50,9 \%)$ foram homens. A idade variou de 3 meses a 86 anos na época do acidente. Além disso, a faixa etária adulta (entre 20 a 59 anos de idade) correspondeu a $114(38,9 \%)$ acidentes. Em relação a cor da pele, $127(43,3 \%)$ se autorreferiram pardos.
Os resultados relacionados às características sociais mostram que $165(56,3 \%)$ destes pacientes residiam em zona urbana ou periurbana. Em relação à escolaridade das vítimas, foi possível observar que $50(17 \%)$ declararam possuir 3 anos completos de estudo ou menos (Tabela 1).

Sobre o animal peçonhento envolvido nos acidentes notificados, $235(80,9 \%)$ acidentes foram por escorpiões e $58(19,1 \%)$ serpentes. Dos acidentes ofídicos, os mais frequentes foram o botrópico com $41(70,6 \%)$ casos e o crotálico com $12(20,8 \%)$ (Tabela 2).

A Figura 1 mostra a descrição do número de acidentes ocorridos em cada mês do período de observação. O período de dezembro de 2016 a março de 2017 foram os de maior incidência em casos de escorpionismo e ofidismo, sendo janeiro de 2017 o mês com maior número de acidentes notificados para estas duas condições (37 e 16 casos, respectivamente).

Tabela 1. Características sociodemográficas das vítimas de acidentes por animais peçonhentos atendidos no município de Vitória da Conquista-BA, no período de julho de 2016 a junho de 2017.

\begin{tabular}{|c|c|c|}
\hline Características & $\mathbf{N}$ & $\%$ \\
\hline \multicolumn{3}{|l|}{ Sexo } \\
\hline Masculino & 149 & 50,9 \\
\hline Feminino & 144 & 49,1 \\
\hline \multicolumn{3}{|l|}{ Faixa etária (anos) } \\
\hline Criança (0 a 10) & 91 & 31 \\
\hline Adolescente (11 a 19) & 40 & 13,7 \\
\hline Adulto (20 a 59) & 114 & 38,9 \\
\hline Idoso $(>60)$ & 48 & 16,4 \\
\hline \multicolumn{3}{|l|}{ Cor da pele } \\
\hline Branca & 33 & 11,3 \\
\hline Amarela & 1 & 0,3 \\
\hline Parda & 127 & 43,3 \\
\hline Preta & 31 & 10,6 \\
\hline Não definida/não declarada & 101 & 34,5 \\
\hline \multicolumn{3}{|l|}{ Zona de residência } \\
\hline Zona urbana ou periurbana & 165 & 56,3 \\
\hline Zona rural & 124 & 42,3 \\
\hline Não definida/não declarada & 4 & 1,4 \\
\hline \multicolumn{3}{|l|}{$\begin{array}{l}\text { Escolaridade (anos completos de } \\
\text { estudo) }\end{array}$} \\
\hline 0 a 3 & 50 & 17 \\
\hline 4 a 7 & 20 & 6,9 \\
\hline 8 a 10 & 10 & 3,4 \\
\hline$>11$ & 12 & 4,1 \\
\hline Não se aplica* & 61 & 20,8 \\
\hline Ignorado/não informado & 140 & 47,8 \\
\hline
\end{tabular}


Tabela 2. Características epidemiológicas acidentes por animais peçonhentos atendidos no município de Vitória da Conquista-BA, no período de julho de 2016 a junho de $2017(\mathrm{~N}=293)$.

\begin{tabular}{|c|c|c|}
\hline Variáveis & $\mathbf{N}$ & $\%$ \\
\hline \multicolumn{3}{|l|}{ Tipo de acidente } \\
\hline Ofidismo & 58 & 19,9 \\
\hline Escorpionismo & 235 & 80,1 \\
\hline \multicolumn{3}{|l|}{ Tipo de acidente ofídico } \\
\hline Botrópico & 41 & 70,6 \\
\hline Crotálico & 12 & 20,8 \\
\hline Laquético & 1 & 1,7 \\
\hline Elapídico & 1 & 1,7 \\
\hline Por serpente não peçonhenta & 1 & 1,7 \\
\hline Serpente não identificada & 2 & 3,5 \\
\hline \multicolumn{3}{|l|}{ Localidade de ocorrência } \\
\hline Vitória da Conquista & 249 & 85 \\
\hline Cidades circunvizinhas & 44 & 15 \\
\hline \multicolumn{3}{|l|}{$\begin{array}{l}\text { Zona de ocorrência em relação } \\
\text { ao tipo de acidente }\end{array}$} \\
\hline \multicolumn{3}{|l|}{ Ofidismo } \\
\hline Zona urbana ou periurbana & 3 & 5,2 \\
\hline Zona rural & 55 & 94,8 \\
\hline \multicolumn{3}{|l|}{ Escorpionismo } \\
\hline Zona urbana ou periurbana & 154 & 65,5 \\
\hline Zona rural & 72 & 30,7 \\
\hline Não definida/não declarada & 9 & 3,8 \\
\hline \multicolumn{3}{|l|}{$\begin{array}{l}\text { Tempo decorrido entre a picada } \\
\text { e o atendimento (horas) }\end{array}$} \\
\hline $0 \mathrm{a} 1 \mathrm{~h}$ & 81 & 27,6 \\
\hline$>1 \mathrm{~h}$ até $3 \mathrm{~h}$ & 110 & 37,5 \\
\hline$>3 \mathrm{~h}$ até $6 \mathrm{~h}$ & 51 & 17,4 \\
\hline$>6 \mathrm{~h}$ até $12 \mathrm{~h}$ & 21 & 7,2 \\
\hline$>12 \mathrm{~h}$ até $24 \mathrm{~h}$ & 8 & 2,7 \\
\hline$>24 \mathrm{~h}$ & 7 & 2,4 \\
\hline Ignorado & 15 & 5,2 \\
\hline \multicolumn{3}{|l|}{ Local da picada } \\
\hline Cabeça & 7 & 2,4 \\
\hline Braço/antebraço & 20 & 6,8 \\
\hline Mão/dedo da mão & 96 & 32,8 \\
\hline Tronco & 12 & 4,1 \\
\hline Coxa/perna & 32 & 10,9 \\
\hline Pé/dedo do pé & 120 & 41,0 \\
\hline Ignorado/não relatado & 6 & 2 \\
\hline
\end{tabular}

Em relação a localização de ocorrência, os acidentes por animais peçonhentos ocorreram em 21 municípios diferentes da região sudoeste da Bahia, sendo que 249 (85\%) foram na cidade de Vitória da Conquista (Tabela 2). Em relação à zona de ocorrência, foi possível observar que 55 $(94,8 \%)$ acidentes ofídicos ocorreram em zona rural, enquanto 154 (65,5\%) acidentes escorpiônicos ocorreram em zona urbana ou periurbana. Constatou-se diferença significativa na distribuição da zona de ocorrência em relação ao tipo de acidente $(\mathrm{p}<0,05)$.

Em $110(37,5 \%)$ dos casos notificados, o atendimento ocorreu mais de uma hora e em até 3 horas após o acidente com o animal. As extremidades pé/dedo do pé, 120 (41\%), e mão/dedo da mão, 96 (32,8\%), foram os locais de picada mais frequentes (Tabela 2).

Quanto aos sinais e sintomas clínicos, 270 $(92,2 \%)$ pacientes apresentaram manifestações locais e destes, 268 (99,2\%) apresentaram dor local. Em 54 (18,4\%) dos casos totais, os pacientes tiveram manifestações sistêmicas. Em relação à gravidade dos acidentes ocorridos, 203 (69,3\%) foram classificados como leves, 72 (24,6\%) como moderados e 13 (4,4\%) como graves (Tabela 3). A gravidade do acidente se mostrou associada a um menor tempo decorrido entre o acidente e $o$ atendimento $(p=0,01)$. Sobre tratamento, em $200(68,5 \%)$ dos casos foi prescrita soroterapia para estes pacientes. Em relação aos desfechos, $239(81,6 \%)$ dos casos evoluíram com cura (Tabela 3$)$.

As soroterapias prescritas foram julgadas inadequadas em 172 (59,7\%) dos atendimentos. Do total de tratamentos inadequados, o uso de número de ampolas acima do indicado foi mais frequente $(124 ; 72,1 \%)$, somando um total de 323 ampolas prescritas a mais do que o recomendado nos protocolos (Tabela 4). Constatou-se diferença significativa na distribuição do tipo de inadequação em relação ao tipo de acidente $(\mathrm{p}<0,01)$. Acidentes ofídicos tiveram mais inadequações do tipo "uso de ampolas inferior ao recomendado" enquanto as inadequações de soroterapia em acidentes escorpiônicos estão mais associadas ao uso de ampolas superior ao recomendado nos protocolos.

\section{Discussão}

Os resultados apresentados mostraram que a prescrição inapropriada de soros antivenenos ocorreu na maioria dos usuários atendidos $(59,7 \%)$. Tanto a subdose quanto as sobredoses prescritas têm alta chance de eventos adversos. $\mathrm{O}$ uso racional de medicamentos deve abranger o estabelecimento da real necessidade de uso do produto, assim como formas farmacêuticas, do- 


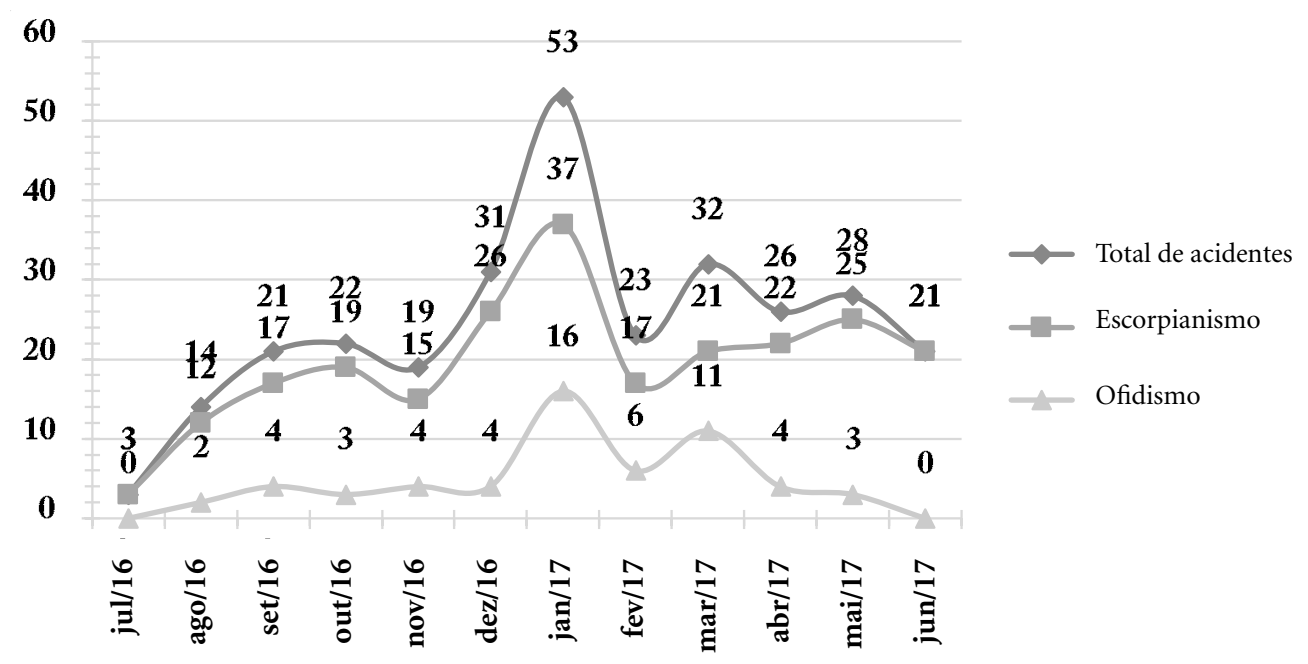

Figura 1. Distribuição do número de acidentes por animais peçonhentos atendidos no município de Vitória da Conquista -BA, no período de julho de 2016 a junho de 2017

ses e período de duração do tratamento adequa$\operatorname{dos}^{31}$.

Apesar da ampla divulgação de diretrizes clínicas, alguns estudos apontam problemas de pouca ou nenhuma adesão a protocolos de tratamento nas mais diversas áreas ${ }^{29,30}$. Outros estudos mostram que para entender a variação de prescrições entre prescritores é preciso conhecer os fatores subjacentes à tomada de decisão destes profissionais ${ }^{32-34}$. A alta rotatividade de médicos da unidade, a incipiência de materiais de consulta, eventuais falhas na divulgação dos protocolos e a ausência de capacitações regulares sobre diagnóstico e tratamento dos acidentes por animais peçonhentos podem ser citadas como fatores que podem influenciar essas prescrições no hospital de estudo. Além disso, a Nota Informativa do Ministério da Saúde sobre a abordagem ao tratamento em casos de acidentes por serpentes do gênero Bothrops e por escorpiões ${ }^{28}$, em situação de escassez de antivenenos, foi publicada em período muito próximo ao período de observação do estudo.

É preciso lembrar que a prescrição médica é um documento complexo que orienta sobre o plano terapêutico para cada paciente. No processo de medicação as responsabilidades devem ser compartilhadas e a tomada de decisão apoiada pelas demais áreas da saúde ${ }^{34}$. A efetiva inserção de uma equipe multiprofissional de urgência/ emergência no atendimento a vítimas por animais peçonhentos poderia ser uma medida para diminuir as inadequações apontadas nos nossos dados, uma vez que vários trabalhos mostram que profissionais, como farmacêuticos e enfermeiros podem atuar de maneira colaborativa ao prescritores neste sentido ${ }^{35-37}$.

Em estudo realizado na Austrália que avaliou o impacto da atuação de farmacêuticos clínicos na emergência de um hospital de médio porte demonstrou que estes profissionais contribuíram com revisão das prescrições médicas, intervenções medicamentosas e orientações da equipe sobre medicamentos, ações estas que poderiam diminuir a prescrição e a administração inadequada de soros antivenenos e demais terapias utilizadas na unidade ${ }^{37}$. Revisão sistemática sobre a atuação destes profissionais também demonstrou estas vantagens e, adicionalmente, concluiu que havia redução de custos e desperdícios hospitalares associada a esta atuação $0^{36}$.

Observou-se que dentre as inadequações encontradas no presente estudo, a mais frequente foi o uso de número de ampolas superior ao recomendado pelos protocolos nacionais $(72,1 \%$ das inadequações foram classificadas em sobredose). A sobredose de medicamentos pode expor pacientes às reações adversas decorrentes do uso destes soros - reações de caráter anafilático ou anafilactoide, precoces ou tardias como a Doença 
Tabela 3. Características clínicas dos acidentes por animais peçonhentos atendidos no município de Vitória da Conquista-BA, no período de julho de 2016 a junho de $2017(\mathrm{~N}=293)$.

\begin{tabular}{|c|c|c|}
\hline Variáveis & $\mathbf{N}$ & $\%$ \\
\hline $\begin{array}{l}\text { Apresentaram sinais e sintomas } \\
\text { locais }^{\star *}\end{array}$ & 270 & 92,2 \\
\hline Dor & 268 & 99,2 \\
\hline Edema & 109 & 40,3 \\
\hline Equimose & 12 & 4,4 \\
\hline Necrose & 1 & 0,3 \\
\hline $\begin{array}{l}\text { Apresentaram sinais e sintomas } \\
\text { sistêmicos }\end{array}$ & 54 & 18,4 \\
\hline $\begin{array}{l}\text { Neuroparalíticas (ptose } \\
\text { palpebral, turvação visual) }\end{array}$ & 19 & 35,5 \\
\hline $\begin{array}{l}\text { Hemorrágicas (gengivorragia, } \\
\text { outros sangramentos) }\end{array}$ & 0 & - \\
\hline Vagais (vômitos, diarréias) & 32 & 59,2 \\
\hline $\begin{array}{l}\text { Miolíticas/hemolíticas } \\
\text { (mialgia, anemia, urina escura) }\end{array}$ & 4 & 7,4 \\
\hline Renais (oligúria/anúria) & 2 & 3,7 \\
\hline \multicolumn{3}{|l|}{ Tempo de coagulaçãoo $o^{\star * *}$} \\
\hline Alterado & 8 & 2,7 \\
\hline Não alterado & 6 & 2,0 \\
\hline Ignorado/não relatado & 283 & 95,3 \\
\hline \multicolumn{3}{|l|}{ Classificação de gravidade ${ }^{*}$} \\
\hline Leve & 203 & 69,3 \\
\hline Moderado & 72 & 24,6 \\
\hline Grave & 13 & 4,4 \\
\hline Ignorado & 5 & 1,7 \\
\hline \multicolumn{3}{|l|}{ Uso de soroterapia* } \\
\hline Sim & 200 & 68,5 \\
\hline Não & 92 & 31,5 \\
\hline \multicolumn{3}{|l|}{ Evolução } \\
\hline Cura & 239 & 81,6 \\
\hline $\begin{array}{l}\text { Óbito por acidentes por } \\
\text { animais peçonhentos }\end{array}$ & 3 & 1,0 \\
\hline Óbito por outras causas & 0 & - \\
\hline Ignorada/desconhecida & 51 & 17,4 \\
\hline
\end{tabular}

do Soro $^{38}$. Além disso, há registros de crises recorrentes de desabastecimento de soros antivenenos alertados pelo Ministério da Saúde seja por adequação e reformas dos laboratórios que os produzem, seja por diminuição da produção ou escassez da matéria-prima ${ }^{28}$. Desta forma, o desperdício de soros antiofídicos e antiescorpiônicos (observada na soma de 323 ampolas prescritas a mais) pode contribuir para a escassez destes medicamentos.
Tabela 4. Análise da adequação da soroterapia prescrita em acidentes por animais peçonhentos atendidos em Vitória da Conquista-BA, no período de julho de 2016 a junho de $2017(\mathrm{~N}=288)$.

\begin{tabular}{lrr}
\hline \multicolumn{1}{c}{ Características } & N & \% \\
\hline $\begin{array}{l}\text { Soroterapia } \\
\quad \text { Adequada }\end{array}$ & 116 & 40,3 \\
$\quad$ Inadequada & 172 & 59,7 \\
$\begin{array}{l}\text { Tipo de inadequação } \\
\quad \text { Uso de ampolas superior ao }\end{array}$ & 124 & 72,1 \\
$\quad \begin{array}{l}\text { indicado } \\
\quad \text { Uso de ampolas inferior ao }\end{array}$ & 44 & 25,6 \\
$\quad \begin{array}{l}\text { indicado } \\
\quad \text { Tipo de soro inadequado }\end{array}$ & 4 & 2,3 \\
$\begin{array}{l}\text { Total de ampolas prescritas a mais } \\
\text { que o indicado nos protocolos }\end{array}$ & 323 & - \\
$\begin{array}{l}\text { Total de ampolas prescritas a } \\
\text { menos que o indicado }\end{array}$ & 91 & - \\
\hline
\end{tabular}

Em contrapartida, constatou-se neste estudo que a soroterapia utilizada para ofidismo está associada significativamente às inadequações do tipo "uso de ampolas inferior ao recomendado". É importante ressaltar que o fato de haver períodos de escassez de soros antiofídicos no tempo de observação na instituição pode ter colaborado com prescrições de número de ampolas inferior ao indicado nas diretrizes. Ainda assim destacase que a subdose é um dos problemas relacionados a medicamentos que pode interferir na efetividade da terapêutica e, por consequência, no desfecho clínico destes pacientes ${ }^{39}$.

As características sociodemográficas da população estudada são semelhantes às de um estudo anterior, realizado em São Paulo que mostrou uma maior frequência vítimas de ofidismo entre homens, adultos em idade funcional ativa, de baixo nível de escolaridade e residentes em área rural $^{10}$. Outros estudos realizados em Goiás ${ }^{40}$, Rio Grande do Norte ${ }^{41}$, Santa Catarina ${ }^{42}$, Minas Gerais $^{43}$ e Amazonas ${ }^{44}$ também obtiveram perfis das vítimas de acidentes por animais peçonhentos similares a esse, assinalando a semelhança destas características nas diferentes regiões brasileiras.

Nossos resultados mostram grande variabilidade etária dos usuários e que todas as faixas etárias estão susceptíveis a estes acidentes. Outros estudos que analisam o perfil sociodemográfico de vítimas destes acidentes também evidenciaram que a maioria delas se encontrava na faixa etária adulta ${ }^{16,25,26}$. Este dado pode ser justificado 
pelo fato deste grupo etário conter os trabalhadores ativos, em especial trabalhadores rurais, com maior chance de encontrar animais peçonhentos durante sua rotina de trabalho ${ }^{26}$.

A sazonalidade dos acidentes por animais peçonhentos nos meses que compreendem o verão foi demonstrada nos dados ora apresentados. Este período de maior incidência de acidentes coincide com os meses mais chuvosos, principalmente aqueles que compreendem o verão, resultado similar ao encontrado em diversos estudos ${ }^{16,25,40,42,45-48}$. Em revisão sistemática sobre as características clínicas e epidemiológicas do escorpionismo, foi registrada uma relação entre acidentes por escorpiões e valores de precipitação pluvial superiores a $30 \mathrm{~mm} / \mathrm{mês}^{7}$. Este fenômeno pode ser explicado pelo fato de que uma vez que a chuva alaga os abrigos de escorpiões, força-os a procurar novos refúgios e transitar pelos ambientes que compartilhamos ${ }^{7,49}$. De forma semelhante, nos meses mais quentes e úmidos o aumento de atividade de trabalhadores rurais os expõem a contato com serpentes ${ }^{25,26}$. Além disso, maiores temperaturas favorecem o acasalamento e reprodução de escorpiões, cobras e aranhas ${ }^{3,44,47,50}$.

Também foi possível observar que a maioria dos acidentes por animais peçonhentos são escorpiônicos e dentre os acidentes ofídicos, o botrópico (causado por jararacas) foi o mais prevalente (70,6\%). Estas frequências são semelhantes a outros estudos epidemiológicos, uma vez que as serpentes do gênero Bothrops têm vasta distribuição geográfica no Brasil, são comuns em vários ecossistemas e tendem a reagir agressivamente quando se sentem ameaçadas ${ }^{9,45,46}$.

Houve diferença estaticamente significante da zona de ocorrência entre ofidismo e escorpionismo, sendo o primeiro mais prevalente em zona rural e o segundo em zona urbana/perirurbana. A zona rural foi a área de maior ocorrência de acidentes ofídicos (94,8\%) visto que as atividades rurais e de caça expõem trabalhadores ao contato com serpentes ${ }^{43,51}$. Os acidentes escorpiônicos, por sua vez, ocorreram em sua maioria na zona urbana/periurbana $(65,5 \%)$, como já demonstrado em outros estudos ${ }^{3,52}$ e deve-se ao fato do ambiente urbano propiciar condições favoráveis para abrigo e proliferação de escorpiões (maiores temperaturas, umidade e presença de lixo $)^{7}$.

Ainda quanto às áreas de ocorrência dos incidentes, foi possível perceber que, durante o período observado, o hospital de estudo recebeu pacientes de 21 cidades diferentes, além de Vitória da Conquista. A diversidade de munícipes é justificada pelo fato desta instituição ser a unidade referência no atendimento de vítimas de acidentes por animais peçonhentos uma vez que a cidade de Vitória da Conquista é região de saúde e referência da macrorregião de 74 cidades vizinhas $^{53}$ (Tabela 5).

A maioria dos acidentes registrados durante o período foi leve $(69,3 \%)$, conforme descrito em outros estudos ${ }^{3,43,46}$. Isso pode estar associado aos curtos intervalos entre a picada e o atendimento. No presente estudo a maioria das vítimas foram atendidas em tempo de até 3 horas após a picada e, como já reportado em literatura, a rapidez no atendimento está diretamente relacionada à evolução do acidente ${ }^{16,26}$.

Por outro lado, apesar da predominância de acidentes leves, na maioria dos casos $(68,3 \%)$ foi prescrita soroterapia e 19,6\% dos usuários evoluíram com manifestações sistêmicas (de maior gravidade que as locais). A mortalidade das vítimas por animais peçonhentos foi de $1 \%$. No entanto, não há como inferir uma possível relação entre o tratamento inapropriado e morte, haja visto que não possuímos dados de comorbidades.

Podemos apontar que as possíveis falhas no preenchimento das fichas de notificação e o fato de não haver variável nestas fichas que contivesse possíveis prescrições adicionais de soros antivenenos, como limitações deste estudo.

É importante dizer que estes dados demonstram a relevância desta causa de morbimortalidade da população de Vitória da Conquista e região e a necessidade que sejam construídas políticas públicas direcionadas a diminuição dos desfechos negativos a partir destes acidentes. Adicionalmente, diante dos resultados de alta frequência de prescrições inadequadas de soros aqui apresentados e discutidos fica evidente a necessidade de que a instituição construa estratégias de forma a subsidiar medidas que assegurem o uso racional deste insumo e controle de seu desperdício.

A inserção da equipe multiprofissional em saúde, em especial de farmacêutico clínico, na unidade de urgência e emergência pode atuar na revisão das prescrições médicas, intervenções relacionadas aos soros antivenenos e orientação da equipe sobre o uso destes medicamentos medidas estas que podem contribuir de maneira significativa na diminuição destas inadequações. 
Tabela 5. Local de ocorrência dos acidentes por animais peçonhentos atendidos no município de Vitória da Conquista-BA, no período de julho de 2016 a junho de $2017(\mathrm{~N}=293)$.

\begin{tabular}{|c|c|c|c|}
\hline Variáveis & $\begin{array}{c}\text { Distância }(\mathrm{km})^{\star} \text { entre } \\
\text { a cidade e Vitória da } \\
\text { Conquista }\end{array}$ & $\begin{array}{l}\text { Número de acidentes } \\
\text { por serpentes }\end{array}$ & $\begin{array}{c}\text { Número de acidentes } \\
\text { por escorpiões }\end{array}$ \\
\hline Vitória da Conquista & - & 29 & 219 \\
\hline \multicolumn{4}{|l|}{ Cidades circunvizinhas } \\
\hline Anagé & 67,3 & 5 & 3 \\
\hline Barra da Estiva & 203 & 2 & - \\
\hline Barra do Choça & 45,4 & 1 & 2 \\
\hline Bom Jesus da Serra & 116 & 2 & - \\
\hline Brumado & 153 & 1 & - \\
\hline Caetanos & 93 & 3 & 2 \\
\hline Caraibas & 96,7 & 1 & 1 \\
\hline Condeúba & 155 & 1 & 1 \\
\hline Encruzilhada & 92,5 & 1 & - \\
\hline Igaporã & 297 & 1 & - \\
\hline Iguaí & 130 & - & 1 \\
\hline Itambé & 50,4 & - & 1 \\
\hline Itororó & 124 & 1 & - \\
\hline Ituaçu & 184 & 3 & - \\
\hline Maetinga & 115 & 1 & 1 \\
\hline Mirante & 160 & 2 & - \\
\hline Piripá & 115 & - & 2 \\
\hline Planalto & 68 & 1 & - \\
\hline Poções & 89,3 & 1 & 1 \\
\hline Ribeirão do Largo & 94,3 & 1 & 1 \\
\hline Tanhaçu & 128 & 1 & - \\
\hline Total & - & 58 & 235 \\
\hline
\end{tabular}

\section{Colaboradores}

CR Santana e MG Oliveira: concepção, delineamento, análise e interpretação dos dados, redação do artigo e aprovação da versão a ser publicada.

\section{Referências}

1. World Health Organization (WHO). Rabies and envenomings. A neglected public health issue: report of a Consultative Meeting. Genebra: WHO; 2007.

2. Chippaux JP. Snake-bites: appraisal of the global situation. Bull World Health Organ 1998; 76(5):515-524.

3. Furtado SS, Belmino JFB, Diniz AGQ, Leite RS. Epidemiology of scorpion envenomation in the state of Ceara, Northeastern BraziL. Rev Inst Med Trop São Paulo 2016; 58:15.

4. Wen FH, Monteiro WM, Silva AMM, Tambourgi DV, Silva IM, Sampaio VS, Santos MC, Sachett J, Ferreira LCL, Kalil J, Lacerda M. Snakebites and scorpion stings in the Brazilian Amazon: identifying research priorities for a largely neglected problem. PLoS Negl Trop Dis 2015; 9(5):e0003701.

5. Chippaux J-P. Epidemiology of envenomations by terrestrial venomous animals in Brazil based on case reporting: from obvious facts to contingencies. $J \mathrm{Ve}$ nom Anim Toxins Incl Trop Dis 2015; 21:13. 
6. Jalali A, Rahim F. Epidemiological review of scorpion envenomation in iran. Iran J Pharm Res IJPR 2014; 13(3):743-756.

7. Santos MSV, Silva CGL, Silva Neto B, Grangeiro Junior CRP, Lopes VHG, Teixeira Junior AG, Bezerra DA, Luna JVCP, Cordeiro JB, Gonçalves Júnior J, Lima MAP. Clinical and Epidemiological Aspects of Scorpionism in the World: A Systematic Review. Wilderness Environ Med 2016; 27(4):504-518.

8. Brasil. Minitério da Saúde (MS). Tecnologia da Informação a Serviço do SUS (DATASUS). Acidentes por animais peçonhentos - notificações segundo tipo de acidente no Brasil em 2016 [página na Internet]. 2016 [acessado 2018 Jan 1]. Disponível em: http://tabnet. datasus.gov.br/cgi/tabcgi.exe?sinannet/cnv/animaisbr.def

9. Leite RS, Targino ITG, Lopes YACF, Barros RM, Vieira AA. Epidemiology of snakebite accidents in the municipalities of the state of Paraíba, Brazil. Cien Saude Colet 2013; 18(5):1463-1471.

10. Bertolozzi MR, Scatena CMC, Franca FOS. Vulnerabilities in snakebites in Sao Paulo, Brazil. Rev Saude Publica 2015; 49:82.

11. Abroug F, Souheil E, Ouanes I, Dachraoui F, Fekih -Hassen M, Ouanes Besbes L. Scorpion-related cardiomyopathy: Clinical characteristics, pathophysiology, and treatment. Clin Toxicol (Phila) 2015; 53(6):511-518.

12. Carmo EA, Nery AA, Jesus CS, Casotti CA. Hospitalizations due to external causes involving contact with animals in a general hospital in the interior of Bahia State, Brazil, 2009-2011. Epidemiol Serv Saude Rev Sist Unico Saude Bras 2016; 25(1):105-114.

13. Monteiro WM, Oliveira SS, Pivoto G, Alves EC, Sachett JAG, Alexandre CN, Fé NF, Guerra MB, Silva IM, Tavares AM, Ferreira LC, Lacerda MV. Scorpion envenoming caused by Tityus cf. silvestris evolving with severe muscle spasms in the Brazilian Amazon. Toxicon 2016; 119:266-269.

14. Saad K, El-Hamed MAA, Abo-Elela MGM, Ahmed AE, Abdel-Baseer KA, Aboul-Khair MD, Metwalley KA, El-Houfey AA, Hasan GM, El-Shareef AM. Neurologic Complications in Children With Scorpionism. J Child Neurol 2017; 32(6):537-542.

15. Albuquerque PLMM, Jacinto CN, Silva Júnior GB, Lima JB, Veras MSB, Daher EF. Acute kidney injury caused by Crotalus and Bothrops snake venom: a review of epidemiology, clinical manifestations and treatment. Rev Inst Med Trop São Paulo 2013; 55(5):295301.

16. Evangelista GF, Azevedo CS. Arachnidism, scorpionism and ophidism in Ouro Preto Municipality, Minas Gerais State, Brazil. Rev Soc Bras Med Trop 2016; 49(6):786-789.

17. Cupo P. Clinical update on scorpion envenoming. Rev Soc Bras Med Trop 2015; 48(6):642-649.

18. Ranaweera GG, Bavanthan V, Nazar ALM, Lokuhetty MDS. Acute renal insufficiency after scorpion sting. Ceylon Med J 2015; 60(1):31-32.

19. Albuquerque PLMM, Silva Júnior GB, Jacinto CN, Lima JB, Lima CB, Amaral YS, Veras MS, Mota RM, Daher EF. Acute kidney injury after snakebite accident treated in a Brazilian tertiary care centre. Nephrology (Carlton) 2014; 19(12):764-770.
20. Cunha FC, Heerdt M, Torrez PPQ, Franca FOS, Molin GZD, Battisti R, Zannin M. First report of hepatic hematoma after presumed Bothrops envenomation. Rev Soc Bras Med Trop 2015; 48(5):633-635.

21. 21Mishra OP, Prasad R. Myocardial dysfunction in children with scorpion sting envenomation. Indian Pediatr 2015; 52(4):291-292.

22. Mahadevan S, Rameshkumar R. Systemic manifestations in children with scorpion sting envenomation: how to manage? Indian J Pediatr 2015; 82(6):497-498.

23. Simon RB. Managing scorpion envenomation in children. Nursing (Lond) 2015; 45(1):13-15.

24. Fundação Ezequiel Dias (FUNED). Bula com informações ao Profissional de Saúde - soro antiescorpiônico. Belo Horizonte: FUNED; 2018.

25. Brasil. Ministério da Saúde (MS). Guia de vigilância em saúde. Brasília: MS; 2014.

26. Brasil. Ministério da Saúde (MS). Manual de diagnóstico e tratamento de acidentes por animais peçonhentos. Guia de vigilância em saúde. Brasília: MS; 2001.

27. Santos R. Contribuição da FUNED para a Produção de Soros Antivenenis e Antitóxicos no Brasil. Rev Min Saude Publica 2005; 6(5):13-19.

28. Brasil. Ministério da Saúde (MS). Portal da Saúde. Nota Informativa: Informações da Coordenação-Geral de Doenças Transmissíveis (CGDT) referentes à nova abordagem ao tratamento em casos de acidentes por serpentes do grupo Bothrops ("jararacas") e por escorpiões, em situação de escassez de antivenenos [página na Internet]. 2016 [acessado 2018 Jan 1]. Disponível em: http://portalsaude.saude.gov.br/index.php/o-ministerio/principal/leia-mais-o-ministerio/197-secretaria-svs/24972-nova-abordagem-ao-tratamento-emsituacao-de-escassez-de-antivenenos

29. Cowie MR, Komajda M. Quality of Physician Adherence to Guideline Recommendations for Life-saving Treatment in Heart Failure: an International Survey. Card Fail Rev 2017; 3(2):130-133.

30. Merchant S, Proudfoot EM, Quadri HN, McElroy HJ, Wright WR, Gupta A, Sarpong EM. Risk factors for Pseudomonas aeruginosa infections consequences of inappropriate initial antimicrobial therapy: a systematic literature review meta-analysis. J Glob Antimicrob Resist 2018; 14:33-44.

31. World Health Organization (WHO). Rational use of drugs. J Fam Welf [periódico na Internet]. 1985 [acessado 2018 Jan 1]; 50:92-94. Disponível em: http://medind.nic.in/jah/t04/s1/jaht04slp92g.pdf

32. Bradley CP. Decision making and prescribing patterns - A literature review. Fam Pract 1991; 8(3):276-287.

33. Bradley CP. Prescribing decisions in general practice-learning and changing. Occas Pap R Coll Gen Pract 1995; 69:10-12.

34. Néri EDR, Viana PR, Campos TA. Dicas Para Uma Boa Prescrição Hospitalar. Fortaleza: Hospital Universitário Walter Cantídio; 2008.

35. Douglass AM, Elder J, Watson R, Kallay T, Kirsh D, Robb WG, Kaji AH, Coil CJ. A Randomized Controlled Trial on the Effect of a Double Check on the Detection of Medication Errors. Ann Emerg Med 2018; 71(1):74-82.

36. Cohen V, Jellinek SP, Hatch A, Motov S. Effect of clinical pharmacists on care in the emergency department: A systematic review. Am J Heal Pharm 2009; 66(15):1353-1361. 
37. Proper JS, Wong A, Plath AE, Grant KA, Just DW, Dulhunty JM. Impact of clinical pharmacists in the emergency department of an Australian public hospital: A before and after study. Emerg Med Australas 2015; 27(3):232-238.

38. Instituto Butantan. Bula profissional - soro antiescorpiônico. São Paulo: Instituto Butantã; 2017.

39. Santos H, Iglésias P, Fernández-Llimós F, Faus MJ, Rodrigues LM. Segundo Consenso de Granada sobre Problemas Relacionados con Medicamentos. Ars Pharm 2004; 43(3-4):175-184.

40. Souza LM, Pinto RNL, Feitosa DT, Júnior Silva NJ. Estudo retrospectivo do escorpionismo no Estado de Goiás. EVS PUC GO 2017; 44:110-114.

41. Tavares AV, Araújo KAM, Marques MRV, Vieira AA, Leite RS. The epidemiology of snakebite in the Rio Grande do Norte State, Northeastern Brazil. Rev Inst Med Trop São Paulo 2017; 59:e52.

42. Quadros RM, Varela AR, Czarin MG, Marques SMT. Acidentes escorpiônicos notificados pelo SINAN na Região Serrana de Santa Catarina, Brasil, 2000-2010. REB 2014; 7(1):96-108.

43. Lima JS, Martelli Júnior H, Martelli DRB, Silva MS, Carvalho SFG, Canela JDR, Bonan PRF. Perfil dos acidentes ofídicos no norte do Estado de Minas Gerais, Brasil. Rev Soc Bras Med Trop 2009; 42(5):561-564.

44. Costa CLSO, Fe NF, Sampaio I, Tadei WP. A profile of scorpionism, including the species of scorpions involved, in the State of Amazonas, Brazil. Rev Soc Bras Med Trop 2016; 49(3):376-379.

45. Albuquerque PLMM, Silva Junior GB, Jacinto CN, Lima CB, Lima JB, Veras MSB, Daher EF. Epidemiological profile of snakebite accidents in a metropolitan area of northeast Brazil. Rev Inst Med Trop São Paulo 2013; 55(5):347-351.

46. Oliveira FN, Brito MT, Cristina I, Morais O, Maria S, Fook L, Albuquerque HN. Accidents caused by Bothrops and Bothropoides in the State of Paraiba: epidemiological and clinical aspects. Rev Soc Bras Med Trop 2010; 43(6):662-667.

47. Coelho JS, Ishikawa EAY, Santos PRSG, Pardal PPO. Scorpionism by Tityus silvestris in eastern Brazilian Amazon. J Venom Anim Toxins Incl Trop Dis 2016; 22(1):24.
48. Leite IC, Valente JG, Schramm JMA, Daumas RP, Rodrigues RN, Santos MF, Oliveira AF, Silva RS, Campos MR, Mota JC. Carga de doença no Brasil e suas regiões, 2008. Cad Saude Publica 2015; 31(7):1551-1564.

49. Clivatti J, Sakata RK, Issy AM. Review of the use of Gabapentin in the Control of Postoperative Pain. Brazilian J Anesthesiol 2009; 59(1):87-98.

50. Superintendência de Controle de Endemias, Secretaria de Estado da Saú de São Paulo. Integrated management for the prevention of proliferation of dengue and leishmaniosis vectors and scorpions. Rev Saude Publica 2007; 41(2):317-320.

51. Santana V, Suchara E. Epidemiologia dos acidentes com animais peçonhentos registrados em Nova Xavantina - MT. Rev Epidemiol Control Infect 2015; 5(3):141-146.

52. Reckziegel GC, Pinto VL. Scorpionism in Brazil in the years 2000 to 2012.J Venom Anim Toxins Incl Trop Dis 2014; 20(1):1-8.

53. Brasil. Ministério da Saúde (MS), SESAB. Regiões de Saúde do Estado da Bahia. Territórios de Identidade [página na Internet]. 2014 [acessado 2017 Set 20]. Disponível em: http://wwwl.saude.ba.gov.br/mapa bahia/Result_Territorioch.asp

Artigo apresentado em 24/02/2018

Aprovado em 27/06/2018

Versão final apresentada em 29/06/2018 